\title{
Modeling of Dirac Electron Tunneling Current in Bipolar Transistor Based on Armchair Graphene Nanoribbon Using a Transfer Matrix Method
}

\author{
Endi Suhendi ${ }^{1}$, Rifky Syariati ${ }^{1}$, Fatimah A. Noor ${ }^{1}$, Neny Kurniasih $^{2}$, Khairurrijal ${ }^{*}$

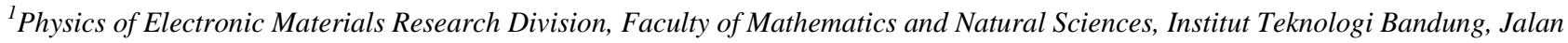 \\ Ganesa 10, Bandung 40132, Indonesia. \\ ${ }^{2}$ Earth Physics and Physics of Complex Systems Research Division, Faculty of Mathematics and Natural Sciences, Institut Teknologi \\ Bandung, Jalan Ganesa 10, Bandung 40132, Indonesia.
}

Received: 18 September 2014 / Accepted: 30 November 2014

\begin{abstract}
:
The Dirac electron tunneling current in an n-p-n bipolar transistor based on armchair graphene nanoribbon (AGNR) has been modeled. The electron wavefunction was derived by employing the relativistic Dirac equation. The transmittance was derived by using the transfer matrix method (TMM). The Landauer formula was used to calculate the Dirac electron tunneling current. The results showed that various variables such as base-emitter voltage, base-collector voltage and the AGNR width affect the Dirac electron tunneling current. It was found that the Dirac electron tunneling current increases with increasing base-emitter and base-collector voltages. Moreover, the increase in the AGNR width results in the increase in the Dirac electron tunneling current.
\end{abstract}

Key words: Armchair graphene nanoribbon, bipolar transistor, dirac electron tunneling current, transfer matrix method

\section{Introduction}

In recent years, graphene nanoribbon (GNR) material has been interesting in research for nanoelectronic devices. Two types of GNR are obtained based on the edge shape: zigzag graphene nanoribbons (ZGNRs) and armchair graphene nanoribbons (AGNRs). The ZGNRs have metal properties for all widths, while the AGNRs can be either semiconducting or metal depending on their widths $[1,2]$. Because of these properties, various nanoelectronic devices can be made [3]. Graphene nanoribbon bipolar transistor is one of the GNR applications for the nanoelectronic devices.

Several models have been done to calculate the tunneling current in bipolar transistor based on silicon and graphene material by solving the Schrödinger equation [4-7]. In this paper, we study the tunneling current in np-n bipolar transistor based on AGNRs by solving the relativistic Dirac equation. The transfer matrix method and Landauer formula are employed to calculate the tunneling current. The effects of base-emitter voltage, base-collector voltage and the AGNR width on the tunneling current will be discussed in detail.

\section{Theoretical Model}

The band structure of AGNRs is given by [8]:

$$
\varepsilon=s \hbar v_{F} \sqrt{k_{z}^{2}+k_{n}^{2}}
$$

where $k_{z}$ is the electron momentum along GNR axis, $k_{n}=n \pi / 3 w$ is the electron transverse momentum which is quantized by the ribbon width, $w$ is the width of AGNRs, $n$ $= \pm 1, \pm 2, \pm 3, \ldots, \quad s=+1$ and $s=+1$ indicate the conduction and valence bands, respectively, $\hbar$ is the reduced Planck constant, and $v_{F}=10^{6} \mathrm{~m} / \mathrm{s}$ is the Fermi velocity. Figure 1 shows the energy diagram of the $n-p-n$ bipolar transistor [4]. The emitter, base and collector are the AGNR doped to be $n, p$ and $n$, respectively. The $p$ - and ntypes of AGNR can be made by doping [9] or electrostatically engineered [10].

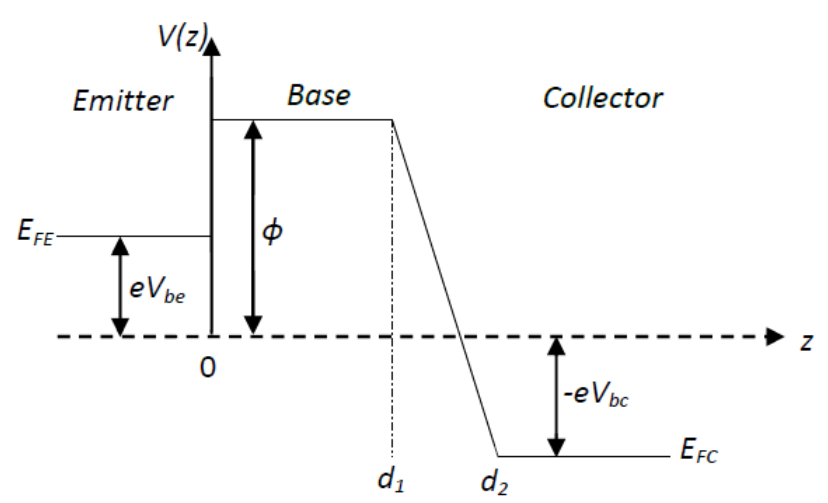

Figure 1. The energy diagram of the n-p-n AGNR with bias.

\footnotetext{
*Corresponding author: Khairurrijal,

E-mail: krijal@itb.ac.id
} 
The electron behavior in graphene is described by the relativistic Dirac-like Hamiltonian as given by [11]

$$
\hat{H}=-i \hbar v_{F} \sigma . \nabla
$$

where $\sigma$ is the Pauli spin matrix. The wave functions in each region are determined by solving Eq. (2).

The electron transmittance is obtained by employing the TMM. In the TMM, the potential profile of the device is divided into many small segments, and the barrier in every segment is assumed constant as shown in Fig. 2 $[12,13]$. The relativistic Dirac equation is solved analytically in every segment. By applying the continuity of the wave function and their derivatives at the boundaries, the transfer matrix between two neighboring segments can be obtained. For all system, the overall transfer matrix is determined by multiplying all these transfer matrices.

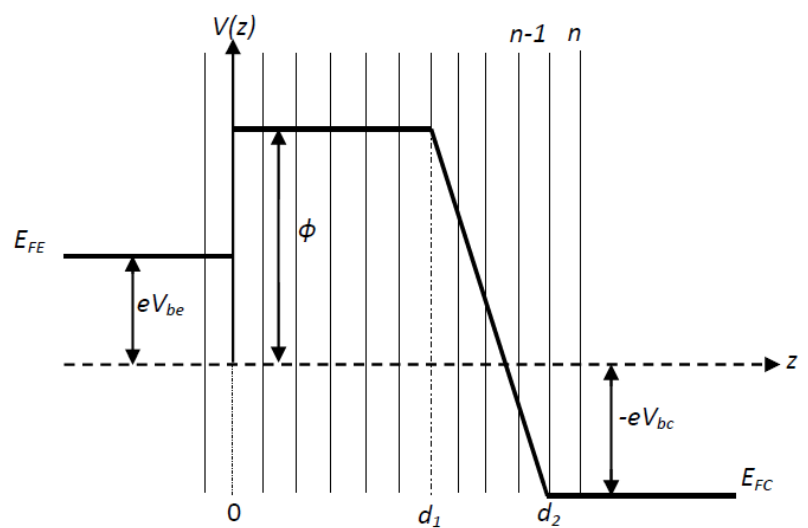

Figure 2. The discretization of potential profile of the $n-p-n$ AGNRs into $n$ segments.

Finally, the Dirac tunneling current is obtained by employing Eq. (3)

$$
I=\frac{2 g_{v} e}{h} \int_{0}^{\infty}\left[f_{E}(E)-f_{C}(E)\right] T(E) d E
$$

where $f_{E}(E)$ and $f_{C}(E)$ are the Fermi-Dirac energy distribution functions for electrons in the emitter and the collector, respectively, $g_{V}$ is the degeneration of GNR $\left(g_{V}=1\right), h$ is the Planck constant, and $T(E)$ is the electron transmittance.

\section{Results and Discussion}

Figure 3 depicts the Dirac tunneling current as a function of base-emitter voltage $\left(\mathrm{V}_{\mathrm{be}}\right)$ for various base-collector voltages $\left(\mathrm{V}_{\mathrm{bc}}\right)$. The AGNRs length as base in the device, $\mathrm{d}_{1}$, the AGNR index (width), $\mathrm{N}$, and the temperature, $\mathrm{T}$, were $25 \mathrm{~nm}, \mathrm{~N}=28(\mathrm{w} \approx 3.32 \mathrm{~nm})$, and $300 \mathrm{~K}$, respectively. It is shown that the Dirac tunneling current is proportional to the base-emitter voltage and it increases with the increase in the base-collector voltage. It is also seen that the difference between the Dirac tunneling current for base-collector voltage more than $0.3 \mathrm{~V}$ is not significant. The increase in the forward bias in $\mathrm{V}_{\mathrm{be}}$ and $\mathrm{V}_{\mathrm{bc}}$ (also called the active mode) causes the increase in Dirac tunneling current [14].

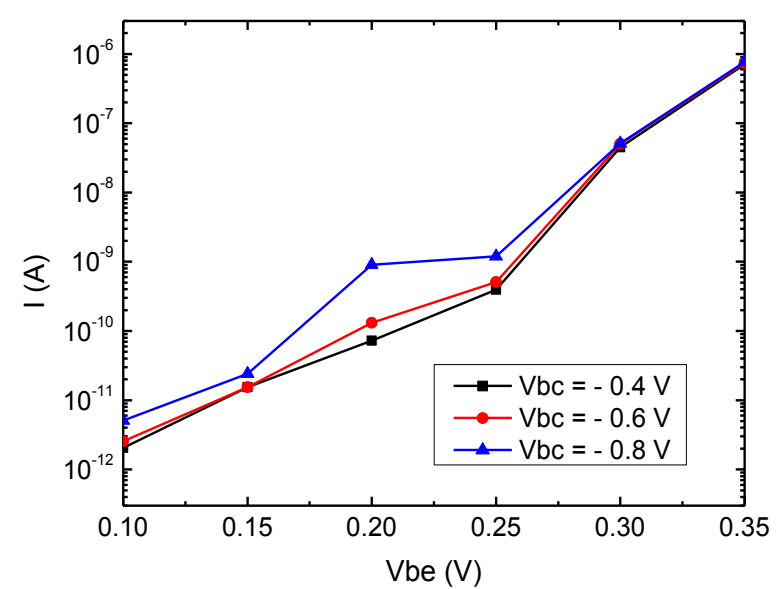

Figure 3. The calculated Dirac tunneling currents as a function of base-emitter voltage for different base-collector voltage.

Figure 4 shows the calculated Dirac tunneling currents as a function of base-collector voltage for various baseemitter voltages. The negative sign in Vbe indicates the reverse bias. It can be seen that the Dirac tunneling currents increase insignificantly with $\mathrm{V}_{\mathrm{bc}}$ for $\mathrm{V}_{\mathrm{be}}$ more than $0.3 \mathrm{~V}$. However, the Dirac tunneling currents increase significantly for $\mathrm{V}_{\mathrm{be}}$ less than $0.3 \mathrm{~V}$ and $\mathrm{V}_{\mathrm{bc}}$ more than $0.7 \mathrm{~V}$. These results show that the $\mathrm{V}_{\mathrm{be}}$ plays an important role in increasing the tunneling current compared to the $\mathrm{V}_{\mathrm{bc}}$.

The Dirac tunneling current as a function of base-emitter voltage for various AGNR index (width) $\mathrm{N}$ is given in Fig. 5. It was taken that the base-collector voltage and the temperature were $0.3 \mathrm{~V}$ and $300 \mathrm{~K}$, respectively. It is shown that the Dirac tunneling current increases with the AGNR index N. The AGNR width $\mathrm{w}$ is proportional to the AGNR index N [14]. It is also shown that the band gap decreases as the AGNR index increases [1]. The results are consistent with those obtained in Ref. $[13,16]$.

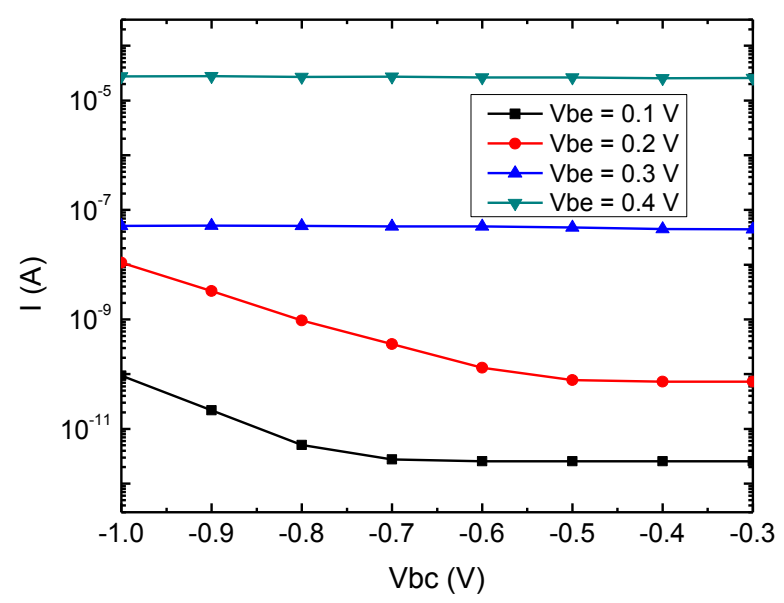

Figure 4. The calculated Dirac tunneling currents as a function of base-collector voltage for different base-emitter voltage.

Figure 6 demonstrates how the base-collector voltage affects to the Dirac tunneling current for various the AGNR index. The base-emitter voltage of $0.3 \mathrm{~V}$ and temperature of $300 \mathrm{~K}$ are used. It is shown that the Dirac 
tunneling current increases insignificantly as the basecollector voltage increases for the same of the AGNR index. As the AGNR index increases, the Dirac tunneling current increases significantly.

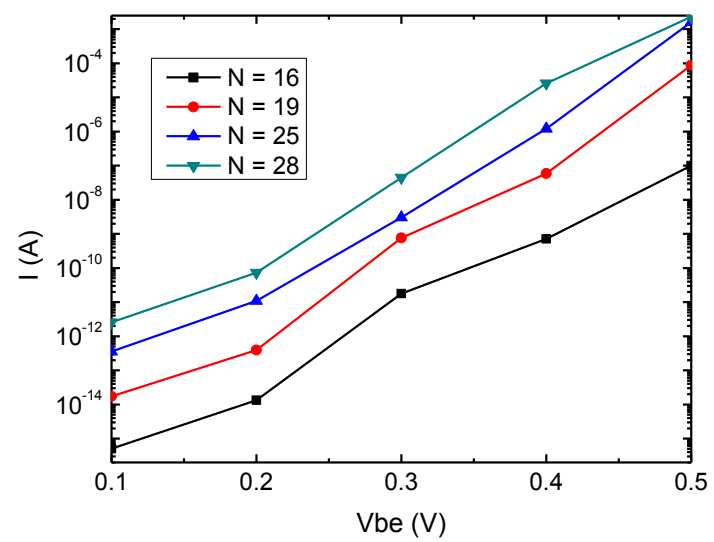

Figure 5. The calculated Dirac tunneling currents as a function of base-emitter voltage for different AGNRs width-N index.

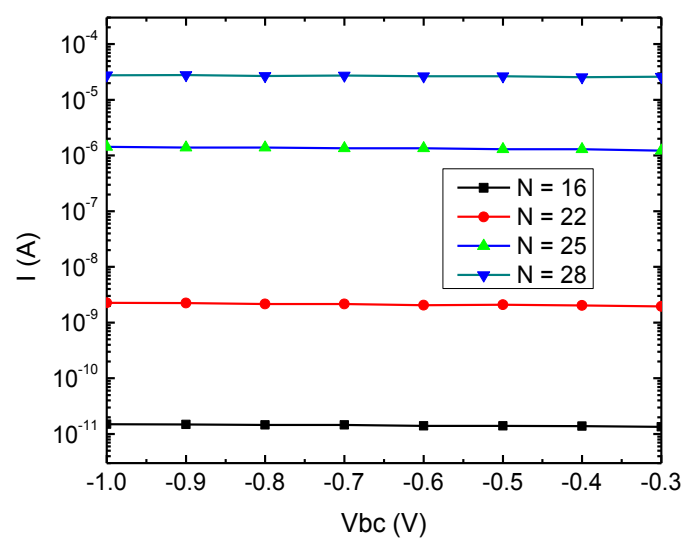

Figure 6. The calculated Dirac tunneling currents as a function of base-collector voltage for different AGNRs width-N index.

\section{Conclusions}

The Dirac electron tunneling current of n-p-n bipolar transistor based on AGNR has been studied by solving the relativistic Dirac-like Hamiltonian. The transmittance was calculated numerically by using the TMM and the Dirac electron tunneling current was obtained by employing the Landauer formula. It is shown that the Dirac tunneling current increases as the base-emitter and base-collector voltages increase. It has been also shown that the Dirac electron tunneling enhances with increasing the AGNR width.

\section{Acknowledgement}

This work was financially supported by "Riset \& Inovasi KK" and "Hibah Kompetensi" Research Grants in the fiscal year 2014.

\section{References}

[1] L. Brey and H. A. Fertig, Electronic states of graphene nanoribbons, Phys. Rev. B, 73, 2006, 235411-1/5.

[2] Y.W. Son, M.L. Cohen, and S.G. Louie, Energy gaps in graphene nanoribbons, Phys. Rev. Lett., 97(21), 2006, 216803-1/4

[3] B. Huang, Q. Yan, Z. Li, and W. Duan, Towards graphene nanoribbon-based electronics, Front. Phys. China, 4, 2009, 269-279.

[4] L. Hasanah, M. Abdullah, Sukirno, T. Winata, and Khairurrijal, Model of a tunneling current in an anisotropic $\mathrm{Si} / \mathrm{Si}_{1-\mathrm{x}} \mathrm{Ge}_{\mathrm{x}} / \mathrm{Si}$ heterostructure with a nanometer-thick barrier including the effect of parallel-perpendicular kinetic energy coupling. Semicond. Sci. Technol., 23, 2008, 125024-1/6.

[5] K. Nadda and M.J. Kumar, Schottky collector bipolar transistor without impurity doped emitter and base: Design and Performance, IEEE Trans. on Electron Devices, 60, 2013, 2956-2959.

[6] G.M. Khanduri and B.S. Panwar, Improved current gain at high collector current densities for $\mathrm{SiGe}$ heterostructure transistors, J. Indian Inst. Sci., 85, 2005, 131-139.

[7] B. Gharekhanlou, S. Khorasani, and R. Sarvari, TwoDimensional Bipolar Junction Transistors, Mater. Res. Express, 1 (1), 2014, 015604-1/10.

[8] T. Fang, A. Konar, H. Xing, and D. Jena, Carrier statistics and quantum capacitance of graphene sheets and ribbons, Appl. Phys. Lett., 91, 2007, 092109-1/3.

[9] B. Huang, Q. Yan, G. Zhou, J. Wu, F. Liu, B. L. Gu, and W. Duan, Making a field effect transistor on a single graphene nanoribbon by selective doping, Appl. Phys. Lett., 91(25), 2007, 253122-1/4.

[10] J. Knoch and J. Appenzeller, Tunneling phenomena in carbon nanotube field-effect transistors, Phys. Status Solidi A, 205, 2008, 679-694.

[11] M.I. Katsnelson, K.S. Novoselov, A. K. Geim, Chiral tunnelling and the Klein paradox in graphene, Nature Phys., 2(9), 2006, 620-625.

[12] W.Z. Shangguan, X. Zhou, S.B. Chiah, G.H. See, and K. Chandrasekaran, Compact gate-current model based on transfer-matrix, J. Appl. Phys., 97, 2005, 123709-1/4.

[13] E. Suhendi, R. Syariati, F.A. Noor, N. Kurniasih, and Khairurrijal, Simulation of Dirac tunneling current of an armchair graphene nanoribbon-based p-n junction using a transfer matrix method, Adv. Mater. Res., 974, 2014, 205209.

[14] S.M. Sze and K.Ng. Kwok, Physics of Semiconductor Devices: New Jersey, 2007, $3^{\text {rd }}$ Ed. pp. 243-290.

[15] F. Liu, X. Liu, J. Kang, and Y. Wang, Improved performance of tunneling FET based on hetero graphene nanoribbons, http://www.arxiv.org., 2013, 13123391-1/3.

[16] D. Jena, T. Fang, Q. Zhang, and H. Xing, Zener tunneling in semiconducting nanotube and graphene nanoribbon $\mathrm{p}-\mathrm{n}$ junctions, Appl. Phys. Lett., 93, 2008, 112106-1/3. 